\title{
Manufacture and study of new polystyrene scintillators
}

\author{
V.G. Senchishin, V.L. Vasilchuk, A.Yu. Borisenko, V.N. Lebedev*, A.F. Adadurov*, \\ N.P. Khlapova*,V.D.Titskaja, V.S. Koba, L.E. Pelipyagina, L.A. Miroshnichenko, \\ V.E. Leman, V.N. Osadchenko, N.A. Kluban*, V.G. Shydlovskij, Mitsaj L.I. \\ Institute for Single Crystals, National Academy of Science of Ukraine, 60 Lenin Ave., 310001 Kharkiv, Ukraine, tel. \\ (0572)307969,E-mail:sench@isc.kharkov.com \\ *Kharkov State University, sq. Svobody 4, 310077 Kharkiv, Ukraine
}

\begin{abstract}
The main optical characteristics and radiation hardness of new polystyrene scintillator UPS98GC were studied. The scintillator UPS-98GC was compared to SCSN-81, produced by Kuraray Co. which is often used in high-energy physics experiments. The dependence of scintillator properties on radiation dose rates as well as on total dose values was studied. It is shown that for relatively small dose rates closed to those expected during scintillator lifetime, our UPS98GC does not yield to SCSN-81.
\end{abstract}

Keywords: plastic scintillators, polystyrene, radiation hardness

Paper received 05.10.99; revised manuscript received 12.01.00; accepted for publication 21.03.00.

\section{Introduction}

Polystyrene scintillators (PS) are widely used for ionizing radiation detection in modern science and technology. These scintillators consist of polystyrene matrix and scintillating dopants. PS specific features are relatively high light yield ( $50 \%$ compared to that of antracene) and rather high transparency. This is why these devices are applied in numerous high-energy physics experiments, namely, ATLAS, CMS, LHCb, ALICE, etc.[1-2].

To obtain high levels of collected light yield for given experiment geometry, PS must have high value of these quantity for luminescence characteristics. Besides, a single CMS calorimeter consists of about 1000 tiles are used, therefore their material (PS) must be commercially available and relatively cheap. At the same time PS must be characterized by sufficient radiation hardness for having high level of stability of its characteristics during lifetime. For instance, CMS tiles, located in the place where more severe dose impacts, are expected to survive at doses up to $50 \mathrm{kGy}$ during 10 years.

Depending on particular detector design, light collection could be accomplished by two main ways: either just from the scintillator (bar) end (Fig.1a) or by means of WLH fiber (Fig. 1b) from tile edges. Both these kinds of samples and read out schemes were tested in our study.

\section{Samples}

To satisfy above requirements, we designed and produced new polystyrene scintillator UPS-98GC. The scintillator material was made by means of thermoinitiated radical polymerization in a silicate glass form. p-terphenyl $(1.5 \%)$ and POPOP $(0.02 \%)$ have been used as scintillating dopants. To obtain an optimal value of polystyrene mean molecular mass, temperature regime of the production process was optimized by means of special computational model. To compare main parameters, we also tested scintillators SCSN 81 produced by Kuraray Co. [3].

The scintillator properties were studied for $20 \times 200 \mathrm{~mm}^{2}$ bars and $200 \times 400 \mathrm{~mm}^{2}$ tiles being cut from PS plates of 3 and $4 \mathrm{~mm}$ thickness. First kind samples $\left(20 \times 200 \mathrm{~mm}^{2}\right)$ were used for technical attenuation length (TAL), $L_{T}$, measurements. ${ }^{90} \mathrm{Sr}$ source was discretedly moved along the sample side with $1 \mathrm{~cm}$ step. The photomultiplier (PMT) was optically connected to the sample's end-wall. Its anode current has been measured for different distances $x$ between source and PMT. Experimental data were fitted by exponential function:

$i=i_{0} \cdot \exp \left(-x / L_{T}\right)$

where $i$ is a PMT anode current, source being placed at point $x, i_{0}$ is a constant determined by scintillator light 


\section{V.G. Senchishin et al.: Manufacture and study of new polystyrene scintillators...}
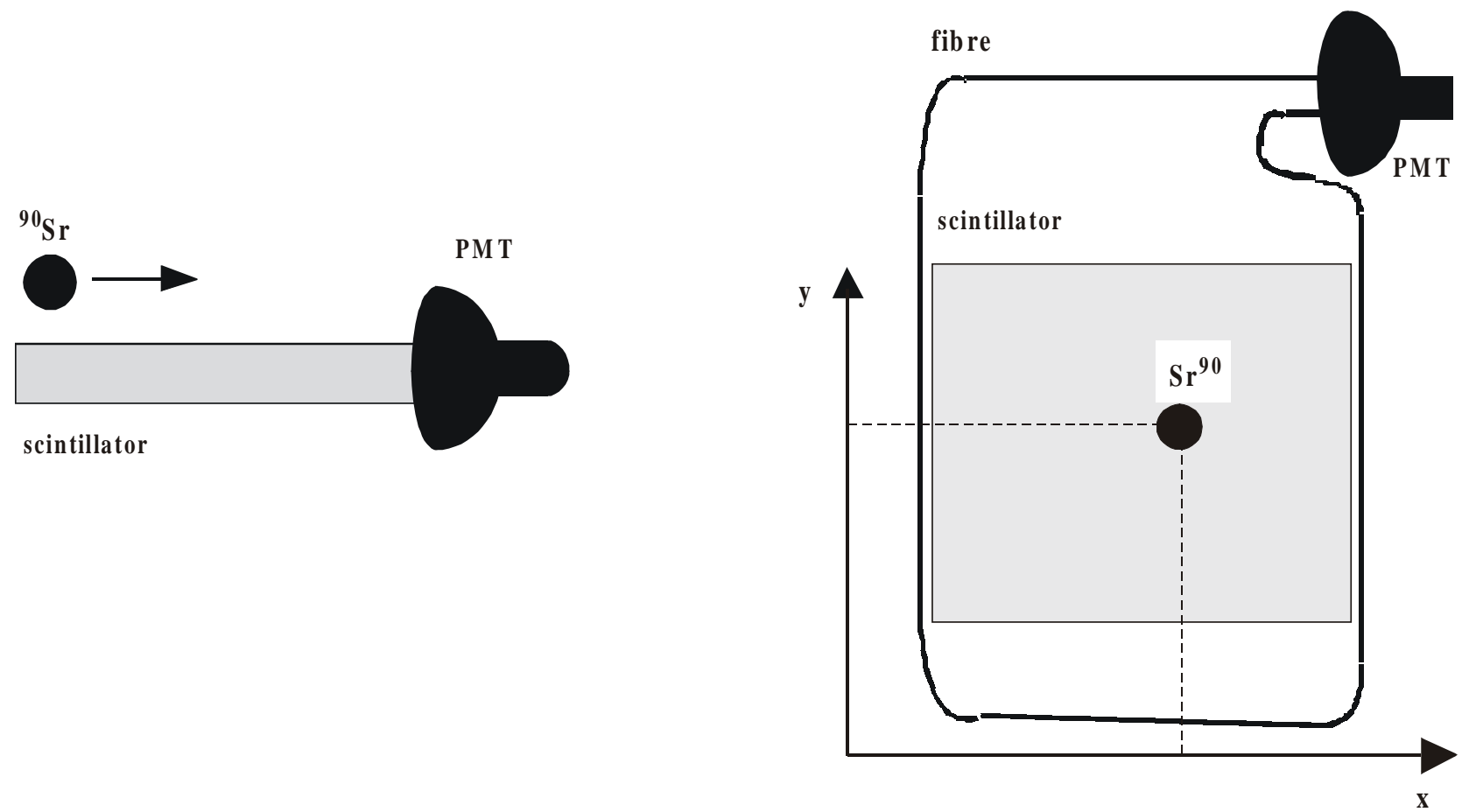

Fig. 1. The setup for polystyrene scintillator characteristic measurements.

yield, $L_{T}$ is a technical attenuation length for PS characteristics luminescent light.

As a light yield value $I$ we took the value of $i$ for $x=1$ $\mathrm{cm}$. This value is close to $i_{0}$. In so doing we obtain an equation with only one free parameter, that makes it possible to calculate $L_{T}$. Another important PS parameter, namely bulk attenuation length, have been measured using cadmium vapor based laser $(\lambda=441 \mathrm{~nm})$. Initial $I_{0}$ and transmitted $\bar{I}$ intensities were used to determine $L$ from the equation:

$$
I=I_{0} \cdot \xi \cdot \exp (-x / L)
$$

were $\xi$ takes into account light reflection by sample surfaces.

Light collection uniformity have been measured using experimental setup similar to that shown in Fig. 1b. Scintillator samples were wrapped by white paper; light was collected by WLS fibers. ${ }^{90} \mathrm{Sr}$-source have been moved along $x$ axis while $y$ was holded constant and equal to 100 mm. Uniformity of light collection have been determined as a mean square deviation of PMT anode current $I(x)$ from its average value $\bar{I}$ according to the equation

$$
r=\frac{\sqrt{\frac{1}{N} \sum(I(x, y)-\bar{I})^{2}}}{\bar{I}}
$$

where $N$ is a number of measurements.

To study the radiation hardness of scintillators being tested, PS samples of $4 \times 20 \times 200 \mathrm{~mm}^{2}$ size were irradiated up to doses of 10-40 kGy. These are typical values that expected to obtain in CMS or LHCb experiments. Samples were irradiated in Kharkov state university using ${ }^{60} \mathrm{Co}$ source, in air at $20^{\circ} \mathrm{C}$ temperature. Exposure dose rates were $0.1-0.25 \mathrm{kGy} /$ hour. As a quantitative test of radiation hardness, the attenuation length $L$ was measured before, during and after (in the course of recovering) irradiation.

\section{Results and conclusions}

The PMT anode currents versus source location for UPS and SCSN-81 scintillator bars $20 \times 200 \mathrm{~mm}^{2}$ are presented in Fig. 2. TAL values, obtained while fitting these data by eq. (1) are compared in Table 1 . The scintillation yield, bulk attenuation length and light collection nonnuniformity are also included in the table. These data show that PS UPS-98GC exceeds SCSN-81 having comparable value of technical attenuation length.

The results of radiation hardness measurements are presented in Fig. 3. It is seen that after irradiation, attenuation length $L$ decreases. This decrease is caused by additional radiation induced absorption because of color centers creation as a result or polystyrene radiative destruction.

Radiation induced light absorption is caused by creation of two kinds of color centers (CC): stable and unstable. Unstable CC are destroyed by oxygen diffusion from air through a scintillator surface. It is a competition of $\mathrm{CC}$ creation and destroying processes that explains the observed dependence of light attenuation length on different dose-rate levels nevertheless resulting in equal total dose [4]. 


\section{V.G. Senchishin et al.: Manufacture and study of new polystyrene scintillators...}

Table 1. Tested parameters of UPS98GC and SCSN-81 scintillators.

\begin{tabular}{|c|c|c|c|c|c|}
\hline PS & Thickness, mm & Light yield, a.u. & $\begin{array}{l}\text { Bulk attenuation } \\
\text { length } L_{0}, \mathrm{~cm}\end{array}$ & $\begin{array}{l}\text { Technical attenuation } \\
\text { length } L_{T}, \mathrm{~cm}\end{array}$ & $\begin{array}{l}\text { Nonuniformity } \\
\text { of } 200 \times 400 \mathrm{~mm}^{2} \text { tile, } \%\end{array}$ \\
\hline UPS-98GC & 3 & 48 & 95 & 55 & 1.61 \\
\hline UPS-98GC & 4 & 65 & 100 & 75 & 2.42 \\
\hline SCSN-81 & 3 & 31 & 98 & 60 & 2.01 \\
\hline SCSN-81 & 4 & 43 & 100 & 75 & 1.40 \\
\hline
\end{tabular}
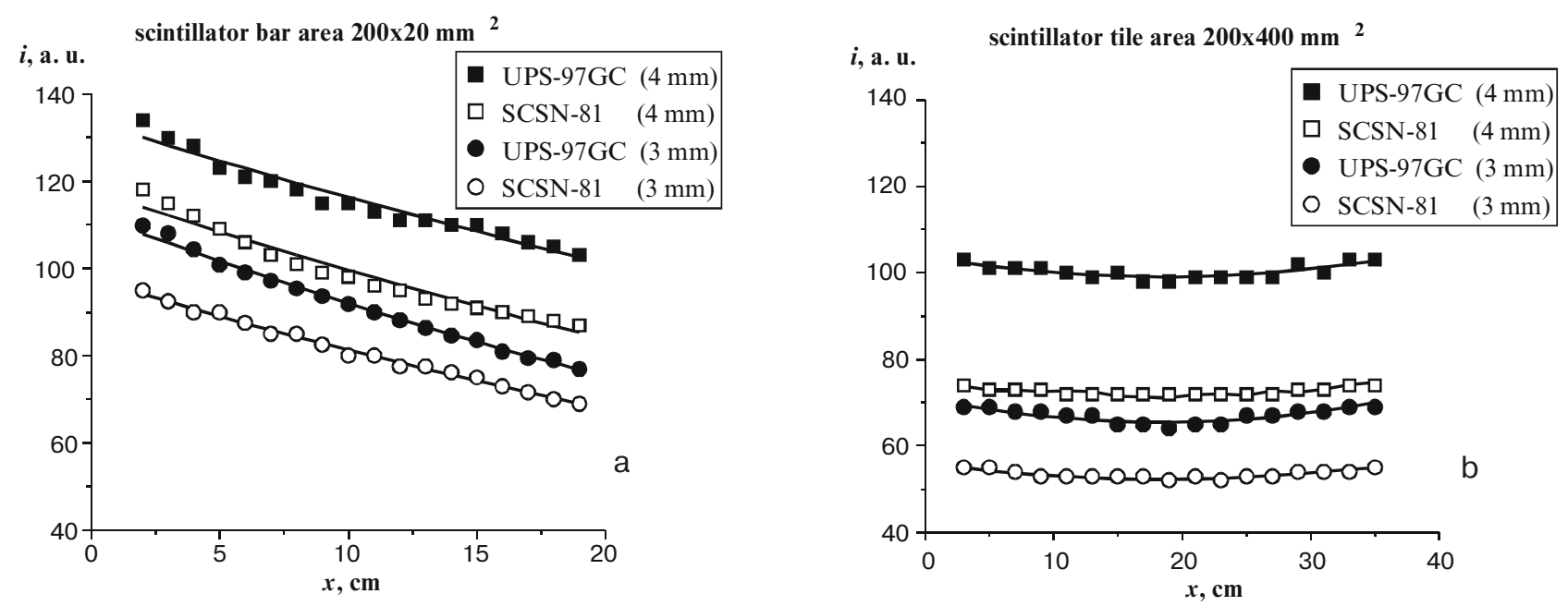

Fig. 2. PMT anode current $v s$. distance to source for bar PS $20 \times 20 \mathrm{~mm}^{2}$ (a) and for middle line of $200 \times 400 \mathrm{~mm}^{2}$ tile (b).
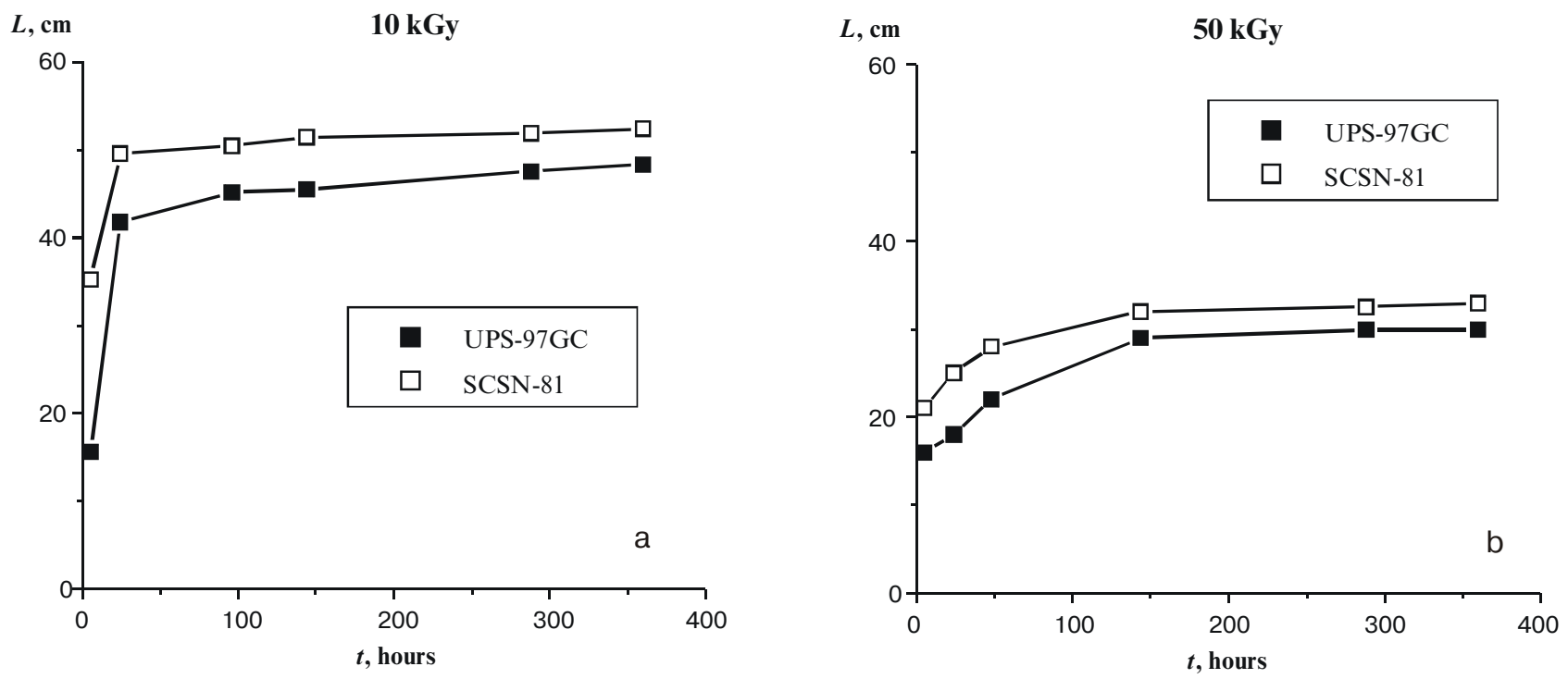

Fig. 3. Bulk attenuation length change under $\mathrm{g}$ - irradiation with dose rate of $0.25 \mathrm{kGy} / \mathrm{h}$ (a) $10 \mathrm{kGy}$ total dose and (b) $50 \mathrm{kGy}$ total dose. 


\section{V.G. Senchishin et al.: Manufacture and study of new polystyrene scintillators...}

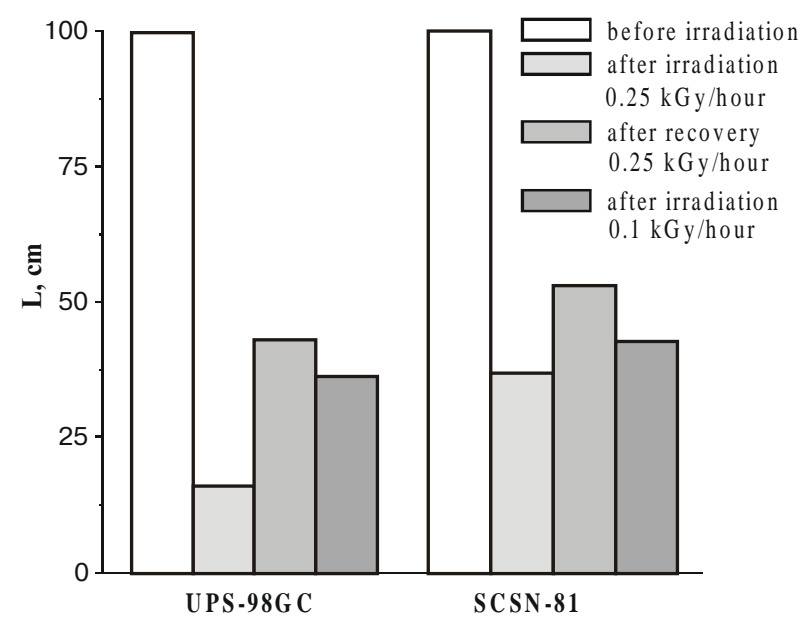

Fig. 4. The scintillator bulk attenuation length change after irradiation of different dose rates.

The production of PS by thermo initiated radical polymerization of scintillation composites in thick layer of 3-4 mm, between polished glass, allows to form a structure from the thermodynamic unstrengthened macro molecules of large length as: $M_{w}=3.5 \cdot 10^{5}$ in the volume and $M_{w}=4 \cdot 10^{5}$ at surfaces. This structure is characterized by the small contents of different defects (molecular end groups, molecular deformation areas), which are centres of destruction and luminescence absorbers formation. This ensures high radiation stability of PS material.
Dose-rate level during scintillator operational lifetime are usually less then those in our test. To bring our results more closely to real operational conditions [1-2], we decrease dose rate by 2.5 times - down to $0.1 \mathrm{kGy} / \mathrm{h}$. Under such a low dose rate (Fig. 4) it happens that PS transparency recovers just in the course of irradiation. When these conditions are satisfied, radiation hardness of our UPS-98GC actually does not yield to that of SCSN81. However, it must be noted that because of special antirad dopants are present, SCSN-81 survives at highest dose-rate levels. This is especially important for scintillators of a large volume where oxygen diffusion processes are very slow.

The results obtained allow to make a conclusion: UPS-98GC scintillators have rather high light yield, transparency, high level of radiation hardness, and can provide sufficient stability of properties for production high energy physics detector tiles of 3 to $4 \mathrm{~mm}$ thickness.

\section{References}

1. ATLAS, Tile Calorimeter, Technical Design Report, CERN/ LHCC 96-42, $334 \mathrm{p}$ (1996).

2. CMS, The Hadron Calorimeter Project. Technical design report, CERN/LHCC 97-31, $451 \mathrm{p}$ (1997).

3. Hasegawa T., Hazumi M., Kasai S.,Tokushuku K., Yamada S., Kojima T., Shimizu T. Tests of new polystyrene-based scintillators //Nucl.Instr. and Meth. A311, pp. 498-504 (1992).

4. Voronkina, O.A. Gunder, G.S. Zhdanov, V.K. Milinchuk, Radiation and photoradiation processes in methyl derivatives of polystyrene and in scintillation compositions based on them //Functional materials, 1, pp. 118-127 (1994). 